J Infect. 2008 November ; 57(5): 385-391. doi:10.1016/j.jinf.2008.08.011.

\title{
ALCOHOL USE AND CLINICAL MANIFESTATIONS OF TUBERCULOSIS
}

\author{
Christina T. Fiske*, \\ Department of Medicine, Duke University Medical Center, Durham, NC, USA 27710
}

Carol D. Hamilton, and

Division of Infectious Diseases and International Health, Department of Medicine, Duke University Medical Center, Durham, NC. USA 27710

Jason E. Stout

Division of Infectious Diseases and International Health, Department of Medicine, Duke University Medical Center, Durham, NC, USA 27710

\section{SUMMARY}

Objectives-Excess alcohol use represents a significant challenge in tuberculosis control. Whether alcohol use enhances transmission of Mycobacterium tuberculosis is not known.

Methods-We analyzed North Carolina, USA surveillance data for all adult (> 14 years) tuberculosis cases reported 1994-2006 $(\mathrm{N}=5,556)$.

Results-The prevalence of excess alcohol use among tuberculosis cases declined from $27.3 \%$ in 1994 to $17.9 \%$ in 2006 . Cases with excess alcohol use were more likely to have pulmonary tuberculosis compared with cases without excess alcohol use (92.5\% vs. $77.2 \%, \mathrm{p}<0.0001)$. Among pulmonary cases, excess alcohol use was associated with cavities on chest radiograph ( $36.8 \%$ vs. $28.2 \%, \mathrm{p}<0.0001)$ and positive acid-fast sputum smears $(65.9 \%$ vs. $45.8 \%$, p <0.0001).

Conclusions-Although excess alcohol use is becoming less prevalent among tuberculosis cases in North Carolina, cases who use excess alcohol had clinical features associated with greater infectiousness, and represent a significant public health problem.

\section{Keywords}

tuberculosis; alcoholism; epidemiology

\section{INTRODUCTION}

Tuberculosis remains an enormous threat to public health worldwide, mainly due to the impact of HIV/AIDS. Over the past decade tuberculosis incidence has declined in the United States from 9.2 cases per 100,000 population in 1994 to 4.4 cases per 100,000 population in 2007 [1]. However, the rate of decline has slowed in recent years, from an average decline

\footnotetext{
Corresponding author/author for reprint requests: Jason E. Stout, Box 3306, Duke University Medical Center, Durham, NC 27710 USA, FAX +1 919681 7494; Phone +1919684 3279; Email stout002@mc.duke.edu.

Current affiliation: Division of Infectious Diseases, Department of Medicine, Vanderbilt University Medical Center, Nashville, TN, USA

Publisher's Disclaimer: This is a PDF file of an unedited manuscript that has been accepted for publication. As a service to our customers we are providing this early version of the manuscript. The manuscript will undergo copyediting, typesetting, and review of the resulting proof before it is published in its final citable form. Please note that during the production process errors may be discovered which could affect the content, and all legal disclaimers that apply to the journal pertain.
} 
of $7.1 \%$ per year during $1993-2000$ to $3.8 \%$ per year during 2001-2007. One potential explanation for this decreased rate of decline is the continued transmission of Mycobacterium tuberculosis among high risk populations. In particular, persons who abuse alcohol and illicit drugs are disproportionately affected by tuberculosis, with reported tuberculosis rates up to 28 fold higher than the age-matched general population [2-4]. Whether excess alcohol use contributes to increased transmission of $M$. tuberculosis is not clear.

We studied the cohort of patients presenting with tuberculosis in the state of North Carolina during 1994 to 2006 to examine the associations between excess alcohol use and clinical manifestations of tuberculosis. We hypothesized that persons with pulmonary tuberculosis and excess alcohol use would be more infectious than other pulmonary tuberculosis cases, as evidenced by having cavitary lung lesions and smear-positive pulmonary tuberculosis, would have lower treatment completion rates, and would require more time to complete tuberculosis treatment than persons who did not use alcohol to excess.

\section{METHODS}

This was a retrospective study of all adult ( $>14$ years old) cases of tuberculosis reported to the North Carolina Tuberculosis Control Program between January 1, 1994 and December 31, 2006. In North Carolina, data from confirmed cases of tuberculosis are routinely recorded by county public health nurses and entered into a central database. Available data elements include the following: demographics (gender, race/ethnicity, age group); occupation (healthcare worker, correctional facility, migrant worker, other, not employed); year of tuberculosis report; human immunodeficiency virus (HIV) status; homelessness during the previous year; residence in a correctional facility or in a long term care facility; excess alcohol use (yes/no/unknown); injection or non-injection illicit drug use (yes/no/ unknown); disease site (pulmonary, extrapulmonary, or both); method of tuberculosis medication administration (self-administered or directly observed therapy); tuberculosis drug resistance (yes/no/unknown); disease verification criteria; history of previous tuberculosis; vital status; and whether tuberculosis treatment was completed. The database also includes clinical information including results of sputum smear and culture, tuberculin skin test results at diagnosis, chest radiograph results, the initial tuberculosis treatment regimen, and the reason for stopping therapy (successful completion, death, lost to followup). Although excess alcohol use is not defined quantitatively, the Centers for Disease Control and Prevention provide general guidelines for public health staff to use when making this assessment. Suggested criteria for classifying tuberculosis cases as having excess alcohol use in the previous 12 months include evidence of participation in an alcohol treatment program, documented medical conditions associated with excess alcohol use (e.g. cirrhosis, pancreatitis), or a diagnosis of alcoholism in medical records.[5] Overall, 192 tuberculosis cases, or 3.5\% of the study population, had missing status for alcohol use. The proportion of tuberculosis cases with missing status for alcohol use declined from $9.7 \%$ in 1994 to $0.6 \%$ in 2006. For purposes of this analysis, subjects with missing or "unknown" status for alcohol use were grouped with persons who did not use excess alcohol.

An anonymised database including all cases reported from 1994 to 2006 was provided to the authors for analysis. The study protocol was approved by the North Carolina State Institutional Review Board and the Duke University Medical Center Institutional Review Board.

\section{Statistical methods}

Prevalence ratios and 95\% confidence intervals were used to describe bivariate associations between excess alcohol use and categorical independent variables. The Cochran-Armitage 
test was used to assess time trends. Because excess alcohol use was prevalent in our population, odds ratios would not accurately reflect the magnitude of risk associated with specific risk factors.[6] Log binomial modeling was therefore performed with SAS PROC GENMOD to estimate prevalence ratios for risk factors associated with excess alcohol use and with potentially more infectious (pulmonary, smear-positive, and cavitary) clinical manifestations of tuberculosis.[7] Unless otherwise specified, subjects with missing data for a given variable were excluded from multivariable models that included that variable. A two-sided p-value of 0.05 was considered statistically significant. All analyses were performed using SAS version 9.1.3 (SAS Institute, Cary, NC).

\section{RESULTS}

Detailed data were available for the 5556 adult tuberculosis cases reported in North Carolina from 1994-2006 (Table 1). Overall, 1326 (23.8\%) tuberculosis cases reported using excess alcohol. Over time, the prevalence of excess alcohol use decreased from $27.3 \%$ in 1994 to $17.9 \%$ in 2006 ( $\mathrm{p}<0.0001$ ) (Figure 1). This decrease was primarily a function of an increasing proportion of foreign-born tuberculosis cases (from 9.6\% in 1994 to 36.9\% in 2006), who had significantly lower rates of excess alcohol use than US-born tuberculosis cases. Male and black tuberculosis cases had significantly higher prevalences of excess alcohol use than female and non-black tuberculosis cases, respectively. The prevalence of excess alcohol use was highest among cases age 25-64, with lower prevalence among younger and older cases. There was significant overlap among excess alcohol use, homelessness, and illicit drug use (Figure 2), but most (677/1326, 51.5\%) tuberculosis cases who used excess alcohol were not homeless and did not use illicit drugs. Tuberculosis cases who used excess alcohol also had a higher prevalence of coinfection with the human immunodeficiency virus (HIV) than cases who did not use excess alcohol. Among tuberculosis cases with known HIV status, $21.1 \%$ who used excess alcohol were HIVseropositive, whereas $12.8 \%$ of tuberculosis cases who did not use excess alcohol were HIVseropositive (Relative risk 1.65, 95\% confidence interval 1.42-1.91).

North Carolina tuberculosis cases with excess alcohol use were more likely to have pulmonary tuberculosis than cases without excess alcohol use (92.5\% of cases with excess alcohol use had pulmonary tuberculosis vs. $77.2 \%$ of cases without excess alcohol use had pulmonary tuberculosis, $\mathrm{p}<0.0001)$. Furthermore, among cases with pulmonary tuberculosis, persons with excess alcohol use were more likely to have cavitary disease noted on chest radiographs than cases without excess alcohol use (36.8\% vs. $28.2 \%$, p<0.0001). Similarly, pulmonary tuberculosis cases with excess alcohol use more often had at least one positive acid-fast sputum smear $(65.9 \%$ vs. $45.8 \%, \mathrm{p}<0.0001)$ and had sputum cultures that grew Mycobacterium tuberculosis (84.6\% vs. $69.5 \%$, p $<0.0001$ ) (Table 2).

Due to small sample size in certain subpopulations (e.g. HIV-positive ages 15-24 or 65+, US born Asian), we were unable to directly adjust for all relevant demographic factors in the entire population of tuberculosis cases. We therefore performed a selective analysis restricted to white and black US-born subjects, who comprised $75 \%$ of tuberculosis cases in North Carolina during this period. After adjustment for sex, age group, racial group, HIV status, and homelessness, tuberculosis cases with excess alcohol use were significantly more likely to have pulmonary tuberculosis (prevalence ratio $1.15,95 \%$ confidence interval 1.11-1.19). Among US-born white or black subjects with pulmonary tuberculosis, subjects with excess alcohol use were also more likely in adjusted analysis to have smear-positive tuberculosis (prevalence ratio 1.24, 95\% confidence interval 1.14-1.34) and cavitary tuberculosis (prevalence ratio 1.23, 95\% confidence interval 1.09-1.40). Given the large number of TB cases with missing HIV status, we repeated the analysis assuming that all 
persons with missing HIV status were HIV-seronegative; this assumption had no significant impact on the results (data not shown).

Tuberculosis treatment completion was documented in $86.9 \%$ (1152/1326) of cases with excess alcohol use compared with $81.5 \%$ (3448/4230) for cases without excess alcohol use $(\mathrm{p}<0.0001)$. However, after adjusting for age the difference in completion rates between cases with excess alcohol use and those without excess alcohol use was not statistically significant (relative risk 1.00, 95\% confidence interval 0.98-1.03). Adjusting for age eliminated the observed difference in completion rates primarily because cases without excess alcohol use were older and more likely to die prior to completion of tuberculosis treatment (10.9\% died prior to completion of treatment vs. $7.9 \%$ of cases with excess alcohol use who died prior to treatment completion). Among tuberculosis cases with drug susceptible pulmonary disease, and who completed a full course of tuberculosis treatment, cases with excess alcohol use required slightly more time to complete treatment (median 6.65 months vs. 6.42 months for cases without excess alcohol use, $p=0.006$ ). Importantly, the proportion of all cases who received all tuberculosis therapy by directly observed therapy (DOT) increased over time, from 29.9\% in 1994 to $83.8 \%$ in 2006. Direct observation of all tuberculosis therapy was particularly associated with higher completion rates among cases with excess alcohol use ( $88.8 \%$ of subjects with excess alcohol use who received directly observed therapy compared with $82.0 \%$ of subjects with excess alcohol use who did not receive directly observed therapy, $\mathrm{p}=0.001$ ).

\section{DISCUSSION}

We found that tuberculosis cases in North Carolina from 1994 to 2006 who reported excess alcohol consumption were more likely to present with pulmonary tuberculosis compared with cases that did not use excess alcohol. Furthermore, among pulmonary tuberculosis cases, persons with excess alcohol use were more likely to have positive acid fast sputum smears and cavitary lesions on chest radiograph, both of which are associated with a greater burden of disease and a greater degree of potentially more infectious tuberculosis [8].

Alcohol use has consistently been associated with increased rates of tuberculosis [2,3,9]. Whether excess alcohol use actually leads to increased transmission of M. tuberculosis is not clear, as previous studies have reported conflicting results. [10-12] A study of 1,250 adults with pulmonary tuberculosis found that excess alcohol use was not independently associated with cavitary lesions in a multivariate analysis [10]. Tobacco use was associated with cavitary lesions in univariate analysis only. These study findings may not be generalizable because the researchers excluded several groups, including HIV seropositive individuals, those patients that could not be located, and those subjects with negative sputum culture for M. tuberculosis. Another study of 105 tuberculosis patients in Taiwan did not find alcoholism associated with smear positivity in an adjusted analysis, and alcohol use was subsequently not included in a smear positive prediction model [12]. Our study included more subjects, and consequently had more power to detect significant differences in the clinical manifestations of tuberculosis in subjects that consumed excess alcohol compared to those that did not. In contrast to the first study, we were primarily interested in the public health impact of excess alcohol use, and therefore evaluated microbiologic markers of infectiousness (sputum smear and culture) in addition to radiographic markers of infectiousness.

There are several explanations for the association between excess alcohol use and more infectious tuberculosis. Persons who consume excess alcohol also frequently smoke tobacco [13]. Smoking has been shown to be associated with pulmonary tuberculosis in previous studies and is a potential confounder.[14,15] For example, Leung, et al performed a large 
case control analysis evaluating the association between smoking and tuberculosis in Hong Kong [16]. Tuberculosis cases that smoked had higher odds of presenting with pulmonary disease, a higher proportion of cavitary lesions on chest radiography and a higher proportion of positive sputum smear and culture. Tobacco use was significantly associated with regular alcohol use (five or more times a week). An additional cross-sectional study from Spain also reported that smoking tobacco was associated with increased pulmonary tuberculosis and cavitary disease [14]. Smoking is associated with other social variables such as homelessness and illicit drug use that may lead to a delay in diagnosis of tuberculosis and more advanced disease at presentation. However, smoking also has known effects on pulmonary host defense such as alteration of the mucociliary apparatus, and perturbation of natural and acquired cell immunity [17], and therefore may have a direct role in advanced lung disease in pulmonary tuberculosis.

Alcohol consumption itself may reduce host resistance to tuberculosis leading to more advanced pulmonary disease at presentation. It is difficult to define the exact mechanisms by which alcohol exerts immunosuppressive effects because excess alcohol use is often associated with other comorbidities such as nutritional deficiencies, liver disease, and cigarette smoking (as mentioned above). Nevertheless, there is clear evidence that alcohol ingestion interferes with anatomic barriers that protect the lung from infection, impairs the actions of cytokines such as TNF-alpha and inhibits the expression of growth factors such as G-CSF in the lung [18]. Alcohol use also interferes with phagocytosis and intracellular killing of mycobacteria by macrophages. These immune deficits could be exacerbated by concomitant nutritional deficiencies or tobacco use $[19,20]$. In addition, alcohol use may modulate the host immune response and predispose to lung cavity formation. Cavitary lung disease in patients with tuberculosis is associated with a predominant T-helper 2 CD4+ cell response in alveolar fluid, and this response in combination with elevated levels of tumor necrosis factor- $\alpha$ (TNF- $\alpha$ ) leads to tissue necrosis and destruction.[21] Alcohol use enhances the T-helper $2 \mathrm{CD} 4+$ response but results in lower secretion of TNF- $\alpha$.

Excess alcohol use may also be associated with more infectious forms of tuberculosis because persons that consume excess alcohol may not seek, or have access to, healthcare when they fall ill, resulting in delayed diagnosis and more advanced disease in the form of cavitary lesions and positive sputum smears [11,12]. There have been numerous reports of tuberculosis outbreaks among the homeless population [22,23], likely due to increased risk of tuberculosis exposure, delays in tuberculosis diagnosis, and limited access to healthcare resources. Studies have shown a higher prevalence of drug and alcohol abuse in homeless tuberculosis cases compared with the non-homeless population with tuberculosis [8]. However, in our study the majority of excess alcohol users did not report homelessness or illicit drug use, indicating that an inability or unwillingness to access healthcare is not the sole explanation for our findings. In addition, because a majority of tuberculosis cases that used excess alcohol did not have these other comorbid conditions, a history of alcohol abuse should be actively solicited in all persons with tuberculosis, not only the homeless or persons who abuse illicit drugs.

Previous studies have shown that concurrent use of alcohol has been associated with delay of diagnosis and initiation of tuberculosis therapy $[24,25]$ as well as noncompliance with tuberculosis treatment [26,27], infection with multi-drug resistant tuberculosis [9], and worse outcomes such as increased mortality [28]. In addition, other studies have found higher risks of treatment interruption and non-adherence to therapy [29,30] in individuals using excess alcohol. We found a higher proportion of cavitary disease, which may have been a surrogate for delayed diagnosis, in tuberculosis cases that reported excess alcohol use. After adjusting for age, completion rates for tuberculosis therapy in North Carolina were similar between tuberculosis cases that used excess alcohol and those that did not, 
although cases that used excess alcohol took a median one week longer to complete therapy. One possible reason for the comparable rates of treatment completion between both groups of tuberculosis patients in our study was that directly observed therapy (DOT) was increasingly employed during our study period. In our population, DOT was associated with higher completion rates among tuberculosis cases who used excess alcohol, whereas cases who did not use excess alcohol had similar completion rates with or without DOT. Our study agrees with previously reported data demonstrating that TB patients receiving DOT had less relapse and less emergence of multi-drug resistant organisms than patients not receiving DOT [31]. DOT is now widely accepted, and should remain an integral part of tuberculosis treatment programs. This is especially true in those cases at risk for default including patients who use excess alcohol or illicit drugs.

Our study demonstrates that racial disparities in tuberculosis continue. We found that black race was the predominant race reported in our study population and represented $54.1 \%$ of all tuberculosis cases. $70.1 \%$ of all tuberculosis cases that reported excess alcohol use in our study were black. In 2007, blacks in the United States had tuberculosis rates 8.3 times greater than whites [1]. In addition, a retrospective analysis of tuberculosis cases in nonHispanic blacks and non-Hispanic whites in seven southeastern states from 1991-2002 [32] found that black cases reported more excess alcohol use compared with whites ( $24 \%$ v. $17 \%$ respectively).

The strengths of our study include its large population based database of all cases of tuberculosis reported to North Carolina during the study period as well as consistent reporting of clinical data such as chest radiograph findings and microbiology results. Our study has several limitations. Despite the large sample size, the dataset had a high proportion of missing data for certain variables, particularly HIV status. Prior work with this dataset suggests that most persons with missing HIV status were at low risk of HIV infection [33], and repeating the analysis including all HIV-unknown cases as HIV-negative had no significant effect on the results.

Another limitation is the subjective nature of the definition of excess alcohol use. Selfreported alcohol use is usually underestimated, rather than overestimated [34].

Misclassification of excess alcohol use is therefore most likely to be in the direction of underreporting, which would most likely have attenuated the strength of any associations rather than creating falsely positive associations among excess alcohol use and the outcomes in this study. However, one must acknowledge the possibility that clinicians may have been biased toward finding a cavity in patients with excess alcohol use. Such a bias is unlikely to explain the higher prevalence of pulmonary disease and positive smear/culture status among TB cases with excess alcohol use. Finally, excess alcohol use may be significantly confounded by cigarette smoking, which as mentioned above, has been associated with increased incidence and severity of tuberculosis disease in several studies $[14,16]$. We were unable to quantify the amount of cigarette smoking in our tuberculosis patient population.

In conclusion, excess alcohol use is associated with markers of more infectious tuberculosis such as cavitary lung disease and positive sputum smears. Excess alcohol use poses a significant barrier to tuberculosis elimination in low-incidence countries such as the United States because it is intertwined with socioeconomic disadvantage and is associated with higher infectious risk to the general public. Studies of interventions that concurrently treat excess alcohol use and tuberculosis are needed to make progress in tuberculosis elimination.

\section{Reference List}

1. Anon. Trends in tuberculosis--United States, 2007. MMWR Morb Mortal Wkly Rep 57:281-285. [PubMed: 18354371] 
2. Feingold AO. Association of tuberculosis with alcoholism. Southern Medical Journal 69:13361337. [PubMed: 982112]

3. Buskin SE, Gale JL, Weiss NS, et al. Tuberculosis risk factors in adults in King County, Washington, 1988 through 1990. American Journal of Public Health 84:1750-1756. [PubMed: 7977912]

4. Friedman LN, Sullivan GM, Bevilaqua RP, et al. Tuberculosis screening in alcoholics and drug addicts. American Review of Respiratory Disease 136:1188-1192.

5. Centers for Disease Control and Prevention. Appendix SUR I RVCT Form Completion Instructions version 1.20. Last updated on 2003 Accessed at http://ftp.cdc.gov/pub/Software/TIMS/Documentation/Apx\%20SUR\%20I\%20RVCT\%20Form $\% 20$ Completion\%20Instructions.pdf on 5-20-2006

6. McNutt LA, Wu C, Xue X, et al. Estimating the relative risk in cohort studies and clinical trials of common outcomes. American Journal of Epidemiology 157:940-943. [PubMed: 12746247]

7. Walker, GA. Common statistical methods for clinical research with SAS examples. 2. Cary, NC: SAS Institute Inc.; 2002.

8. Haddad MB, Wilson TW, Ijaz K, et al. Tuberculosis and homelessness in the United States, 1994-2003. JAMA 293:2762-2766. [PubMed: 15941806]

9. Fleming MF, Krupitsky E, Tsoy M, et al. Alcohol and drug use disorders, HIV status and drug resistance in a sample of Russian TB patients. International Journal of Tuberculosis and Lung Disease 10:565-570.

10. Moran A, Harbour DV, Teeter LD, et al. Is alcohol use associated with cavitary disease in tuberculosis? Alcohol Clin Exp Res 31:33-38. [PubMed: 17207099]

11. Rodrigo T, Cayla JA, Garcia dO, et al. Characteristics of tuberculosis patients who generate secondary cases. International Journal of Tuberculosis and Lung Disease 1:352-357.

12. Wang CS, Chen HC, Chong IW, et al. Predictors for identifying the most infectious pulmonary tuberculosis patient. J Formos Med Assoc 107:13-20. [PubMed: 18218573]

13. True WR, Xian H, Scherrer JF, et al. Common genetic vulnerability for nicotine and alcohol dependence in men. Archives of General Psychiatry 56:655-661. [PubMed: 10401514]

14. Altet-Gomez MN, Alcaide J, Godoy P, et al. Clinical and epidemiological aspects of smoking and tuberculosis: a study of 13,038 cases. International Journal of Tuberculosis and Lung Disease 9:430-436.

15. Bates MN, Khalakdina A, Pai M, et al. Risk of tuberculosis from exposure to tobacco smoke: a systematic review and meta-analysis. Archives of Internal Medicine 167:335-342. [PubMed: 17325294]

16. Leung CC, Li T, Lam TH, et al. Smoking and tuberculosis among the elderly in Hong Kong. Am J Respir Crit Care Med 170:1027-1033. [PubMed: 15282201]

17. Arcavi L, Benowitz NL. Cigarette smoking and infection. Archives of Internal Medicine 164:2206-2216. [PubMed: 15534156]

18. Happel KI, Nelson S. Alcohol, immunosuppression, and the lung. Proc Am Thorac Soc 2:428-432. [PubMed: 16322595]

19. Vander Top EA, Wyatt TA, Gentry-Nielsen MJ. Smoke exposure exacerbates an ethanol-induced defect in mucociliary clearance of Streptococcus pneumoniae. Alcohol Clin Exp Res 29:882-887. [PubMed: 15897734]

20. Vander Top EA, Perry GA, Snitily MU, et al. Smoke exposure and ethanol ingestion modulate intrapulmonary polymorphonuclear leukocyte killing, but not recruitment or phagocytosis. Alcohol Clin Exp Res 30:1599-1607. [PubMed: 16930223]

21. Mazzarella G, Bianco A, Perna F, et al. T lymphocyte phenotypic profile in lung segments affected by cavitary and non-cavitary tuberculosis. Clinical and Experimental Immunology 132:283-288. [PubMed: 12699418]

22. Anon. Tuberculosis transmission in a homeless shelter population--New York, 2000-2003. MMWR Morb Mortal Wkly Rep 54:149-152. [PubMed: 15716807]

23. Anon. Public health dispatch: tuberculosis outbreak among homeless persons--King County, Washington, 2002-2003. MMWR Morb Mortal Wkly Rep 52:1209-1210. [PubMed: 14668713] 
24. Kiwuwa MS, Charles K, Harriet MK. Patient and health service delay in pulmonary tuberculosis patients attending a referral hospital: a cross-sectional study. BMC Public Health 5:122. [PubMed: 16307685]

25. van der Werf MJ, Chechulin Y, Yegorova OB, et al. Health care seeking behaviour for tuberculosis symptoms in Kiev City, Ukraine. International Journal of Tuberculosis and Lung Disease 10:390395.

26. Burman WJ, Cohn DL, Rietmeijer CA, et al. Short-term incarceration for the management of noncompliance with tuberculosis treatment. Chest 112:57-62. [PubMed: 9228358]

27. Santha T, Garg R, Frieden TR, et al. Risk factors associated with default, failure and death among tuberculosis patients treated in a DOTS programme in Tiruvallur District, South India, 2000. International Journal of Tuberculosis and Lung Disease 6:780-788.

28. Sterling TR, Zhao Z, Khan A, et al. Mortality in a large tuberculosis treatment trial: modifiable and non-modifiable risk factors. International Journal of Tuberculosis and Lung Disease 10:542-549.

29. Diel R, Niemann S. Outcome of tuberculosis treatment in Hamburg: a survey, 1997-2001. International Journal of Tuberculosis and Lung Disease 7:124-131.

30. Pablos-Mendez A, Knirsch CA, Barr RG, et al. Nonadherence in tuberculosis treatment: predictors and consequences in New York City. American Journal of Medicine 102:164-170.

31. Weis SE, Slocum PC, Blais FX, et al. The effect of directly observed therapy on the rates of drug resistance and relapse in tuberculosis. New England Journal of Medicine 330:1179-1184.

32. Anon. Racial disparities in tuberculosis--selected southeastern states, 1991-2002. MMWR Morb Mortal Wkly Rep 53:556-559. [PubMed: 15229413]

33. Stout JE, Ratard R, Southwick KL, et al. Epidemiology of human immunodeficiency virus testing among patients with tuberculosis in North Carolina. South Med J 95:231-238. [PubMed: $11846251]$

34. Hoyer G, Nilssen O, Brenn T, et al. The Svalbard study 1988-89: a unique setting for validation of self-reported alcohol consumption. Addiction 90:539-544. [PubMed: 7773116] 


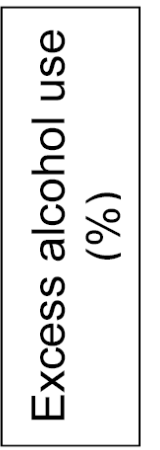

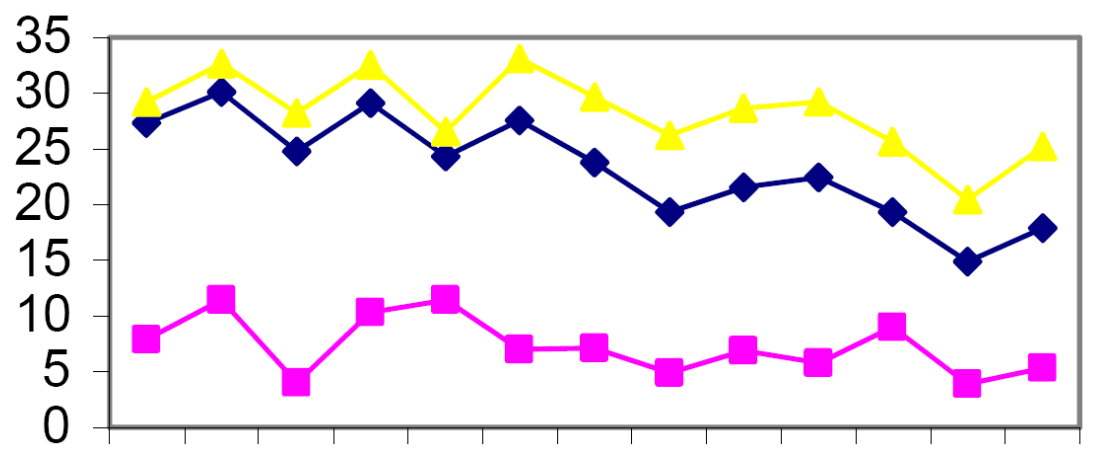

$\multimap$ All TB cases $\rightarrow-$ Foreign born US born

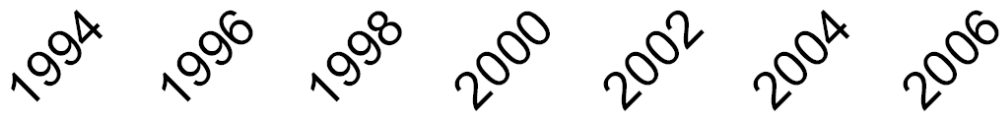

Figure 1.

Excess alcohol use (\%) among tuberculosis cases in North Carolina 1994-2006. 


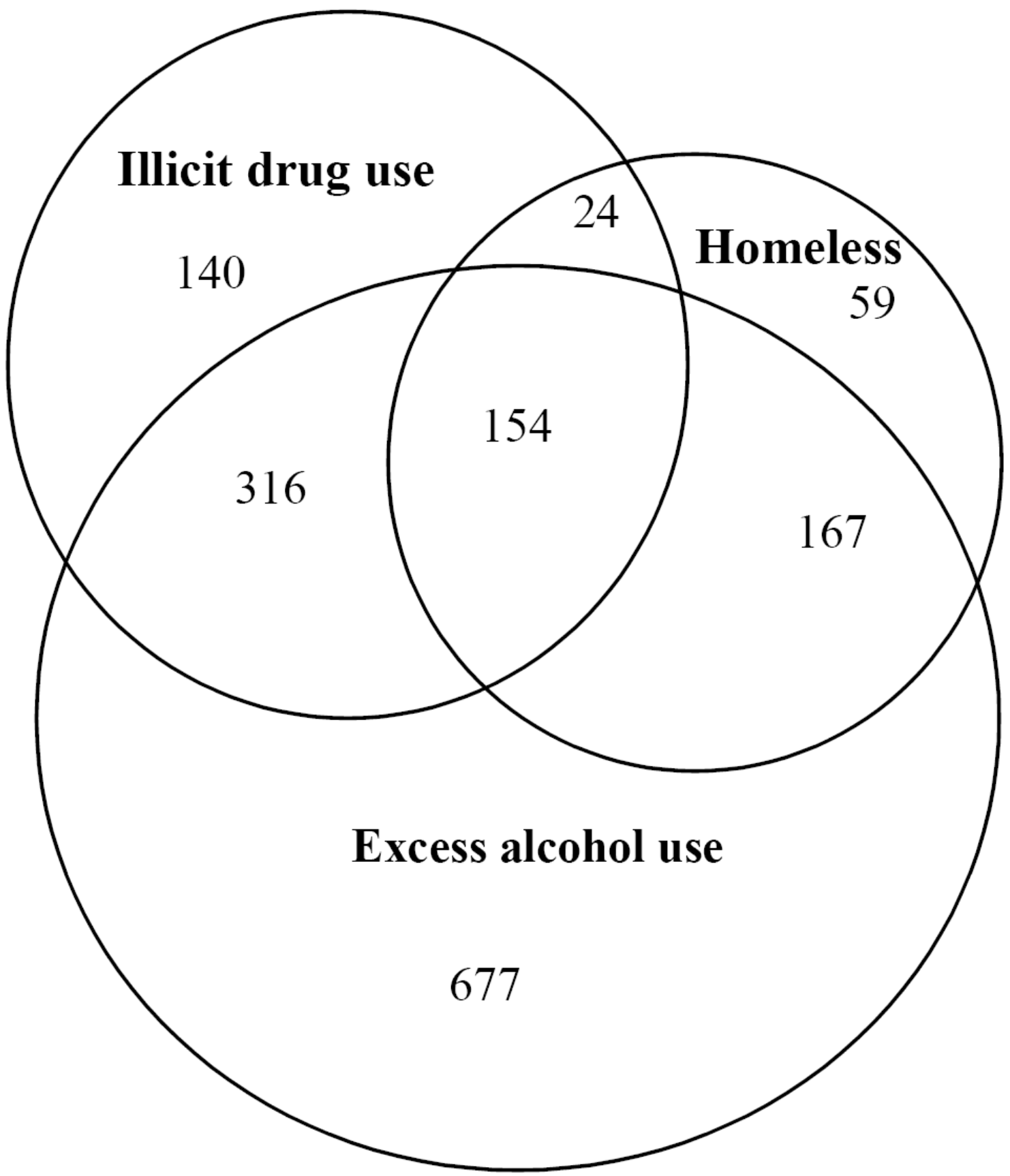

Figure 2.

Overlap among excess alcohol use, illicit drug use (injected or non-injected), and homelessness among tuberculosis cases in North Carolina 1994-2006. Numbers within the circles indicate the number of cases that fit into a given category. Cases missing data on excess alcohol use, illicit drug use, and/or homelessness ( $\mathrm{N}=12$ of the 1326 cases with excess alcohol use) were excluded from the figure. 
Table 1

Conditions associated with excess alcohol use among cases of tuberculosis aged 15 years or older reported in North Carolina 1994-2006 (N=5,556).

\begin{tabular}{|c|c|c|}
\hline Characteristic & $\begin{array}{l}\text { Excess alcohol use (\%) (N=1326 of } 5556 \text { total TB } \\
\text { cases }(23.8 \%))\end{array}$ & $\begin{array}{l}\text { Prevalence ratio ( } 95 \% \text { confidence } \\
\text { interval) }\end{array}$ \\
\hline \multicolumn{3}{|l|}{ Age } \\
\hline $15-24$ & $22 / 423(5.2 \%)$ & 1.0 (referent) \\
\hline $25-44$ & $555 / 1862(29.8 \%)$ & $5.7(3.8-8.7)$ \\
\hline $45-64$ & $646 / 1641(39.4 \%)$ & $7.6(5.0-11.4)$ \\
\hline $65+$ & $103 / 1630(6.3 \%)$ & $1.2(0.8-1.9)$ \\
\hline \multicolumn{3}{|l|}{ Gender } \\
\hline Female & $196 / 1866(10.5 \%)$ & 1.0 (referent) \\
\hline Male & $1130 / 3690(30.6 \%)$ & $2.9(2.5-3.4)$ \\
\hline \multicolumn{3}{|l|}{ Race/Ethnicity by birthplace } \\
\hline \multicolumn{3}{|l|}{ US-born } \\
\hline White & $283 / 1326(21.3 \%)$ & 1.0 (referent) \\
\hline American Indian/Alaskan Native & $18 / 60(30.0 \%)$ & $1.4(0.9-2.1)$ \\
\hline Asian/Pacific Islander & $0 / 3(0 \%)$ & 0 (N/A) \\
\hline Black & $926 / 2840(32.6 \%)$ & $1.5(1.4-1.7)$ \\
\hline Hispanic & $10 / 53(18.9 \%)$ & $0.9(0.5-1.6)$ \\
\hline \multicolumn{3}{|l|}{ Foreign-born } \\
\hline White & $2 / 54(3.7 \%)$ & $0.2(0.0-0.7)$ \\
\hline Asian/Pacific Islander & $5 / 382(1.3 \%)$ & $0.1(0.0-0.1)$ \\
\hline Black & $3 / 165(1.8 \%)$ & $0.1(0.0-0.3)$ \\
\hline Hispanic & $78 / 662(11.8 \%)$ & $0.6(0.4-0.7)$ \\
\hline \multicolumn{3}{|l|}{ HIV status } \\
\hline Negative & $899 / 3233(27.8 \%)$ & 1.0 (referent) \\
\hline Positive & $241 / 585(41.2 \%)$ & $1.5(1.3-1.7)$ \\
\hline Unknown & $186 / 1738(10.7 \%)$ & $0.4(0.3-0.4)$ \\
\hline \multicolumn{3}{|l|}{ Injection drug use } \\
\hline No & $1147 / 5214(22.0 \%)$ & 1.0 (referent) \\
\hline Yes & $83 / 106(78.3 \%)$ & $3.6(3.2-4.0)$ \\
\hline Unknown & $96 / 236(40.7 \%)$ & $1.8(1.6-2.2)$ \\
\hline \multicolumn{3}{|l|}{ Non-injection drug use } \\
\hline No & 785/4711 (16.7\%) & 1.0 (referent) \\
\hline Yes & $466 / 623(74.8 \%)$ & $4.5(4.3-4.9)$ \\
\hline Unknown & $75 / 222(33.8 \%)$ & $2.0(1.7-2.5)$ \\
\hline \multicolumn{3}{|l|}{ Homelessness } \\
\hline No & 993/5094 (19.5\%) & 1.0 (referent) \\
\hline
\end{tabular}

J Infect. Author manuscript; available in PMC 2009 November 1. 


\begin{tabular}{|l|l|l|}
\hline Characteristic & $\begin{array}{l}\text { Excess alcohol use (\%) (N=1326 of 5556 total TB } \\
\text { cases (23.8\%)) }\end{array}$ & $\begin{array}{l}\text { Prevalence ratio (95\% confidence } \\
\text { interval) }\end{array}$ \\
\hline Yes & $\begin{array}{l}\text { (21/424 (75.7\%) } \\
12 / 38(31.6 \%)\end{array}$ & $\begin{array}{l}3.9(3.6-4.2) \\
1.6(1.0-2.6)\end{array}$ \\
Unknown & & \\
\hline Occupation & $11 / 150(7.3 \%)$ & $1.0($ referent $)$ \\
Health Care Worker & $0 / 15(0 \%)$ & $0(\mathrm{~N} / \mathrm{A})$ \\
Correctional facility & $34 / 132(25.8 \%)$ & $3.5(1.9-6.7)$ \\
Migratory Agricultural & $560 / 2202(25.4 \%)$ & $3.5(2.0-6.2)$ \\
Other occupation & $679 / 2926(23.2 \%)$ & $3.2(1.8-5.6)$ \\
Not Employed & $42 / 131(32.1 \%)$ & $4.4(2.3-8.1)$ \\
Not Reported & \multicolumn{2}{|l}{} \\
\hline
\end{tabular}




\section{Table 2}

Differences in disease characteristics between North Carolina tuberculosis cases reported 1994-2006 with and without excess alcohol use. Chest radiographic, sputum smear, and sputum culture data are for cases with pulmonary involvement only.

\begin{tabular}{|l|l|l|l|}
\hline Characteristic & \multicolumn{2}{l|}{ Excess alcohol use } & Prevalence ratio (95\% confidence interval) \\
\hline & Yes & No/Unknown & \\
\hline Site of disease & $1227(92.5 \%)$ & $3266(77.2 \%)$ & $1.20(1.17-1.23)$ \\
Pulmonary (+/- extrapulmonary) & $99(7.5 \%)$ & $964(22.8 \%)$ & \\
Extrapulmonary only & & & \\
\hline Chest radiographic findings & $452(36.8 \%)$ & $920(28.2 \%)$ & $1.31(1.19-1.43)$ \\
Cavitary & $775(63.2 \%)$ & $2346(71.8 \%)$ & \\
Non-cavitary & & & $1.44(1.36-1.52)$ \\
\hline Sputum smear & $809(65.9 \%)$ & $1495(45.8 \%)$ & 1.20 \\
Positive & $418(34.1 \%)$ & $1771(54.2 \%)$ & \\
Negative & $1038(84.6 \%)$ & $2270(69.5 \%)$ & $1.22(1.18-1.26)$ \\
\hline Sputum culture & $189(15.4 \%)$ & $996(30.5 \%)$ & \\
Positive & Negative & &
\end{tabular}

